\title{
Prediction of 6-Month Mortality Using Pre-Extracorporeal Membrane Oxygenation Lactate in Patients with Acute Coronary Syndrome Undergoing Veno-Arterial-Extracorporeal Membrane Oxygenation
}

\author{
Eunchong Kim, M.D. ', Nodirbek Yuldashev Sodirzhon-Ugli, M.D. ', Do Wan Kim, M.D. ', Kyo Seon Lee, M.D.', \\ Yonghwan Lim, M.D. ${ }^{2}$, Min-Chul Kim, M.D., Ph.D. ${ }^{2}$, Yong Soo Cho, M.D. ${ }^{3}$, Yong Hun Jung, M.D., Ph.D. ${ }^{3}$, \\ Kyung Woon Jeung, M.D., Ph.D. ${ }^{3}$, Hwa Jin Cho, M.D., Ph.D. ${ }^{4}$ In Seok Jeong, M.D., Ph.D. ${ }^{1}$ \\ 'Department of Thoracic and Cardiovascular Surgery, Chonnam National University Hospital, Chonnam National University Medical School; ${ }^{2}$ Department of \\ Cardiology, Chonnam National University Medical School; ${ }^{3}$ Department of Emergency, Chonnam National University Hospital, Chonnam National University \\ Medical School; ${ }^{4}$ Department of Pediatrics, Chonnam National University Children's Hospital, Chonnam National University Medical School, Gwangju, Korea
}

\section{ARTICLE INFO}

Received October 13, 2021

Revised December 15, 2021

Accepted December 29, 2021

Corresponding author

In-Seok Jeong

Tel $82-62-220-6546$

Fax 82-62-227-1636

E-mail isjeong1201@gmail.com;

isjeong1201@chonnam.ac.kr

ORCID

https://orcid.org/0000-0002-2249-0667

\begin{abstract}
Background: The effectiveness of extracorporeal membrane oxygenation (ECMO) for patients with refractory cardiogenic shock or cardiac arrest is being established, and serum lactate is well known as a biomarker of end-organ perfusion. We evaluated the efficacy of pre-ECMO lactate for predicting 6-month survival in patients with acute coronary syndrome (ACS) undergoing ECMO.

Methods: We reviewed the medical records of 148 patients who underwent veno-arterial (VA) ECMO for ACS between January 2015 and June 2020. These patients were divided into survivors and non-survivors based on 6-month survival. All clinical data before and during ECMO were compared between the 2 groups.

Results: Patients' mean age was $66.0 \pm 10.5$ years, and 116 (78.4\%) were men. The total survival rate was $45.9 \%(n=68)$. Cox regression analysis showed that the pre-ECMO lactate level was an independent predictor of 6-month mortality (hazard ratio, 1.210; 95\% confidence interval $[\mathrm{Cl}], 1.064-1.376 ; \mathrm{p}=0.004)$. The area under the receiver operating characteristic curve of pre-ECMO lactate was 0.64 ( $95 \% \mathrm{Cl}, 0.56-0.72 ; \mathrm{p}=0.002$; cut-off value $=9.8 \mathrm{mmol} / \mathrm{L})$. Kaplan-Meier survival analysis showed that the cumulative survival rate at 6 months was significantly higher among patients with a pre-ECMO lactate level of $9.8 \mathrm{mmol} / \mathrm{L}$ or less than among those with a level exceeding $9.8 \mathrm{mmol} / \mathrm{L}(57.3 \% \mathrm{vs}$. $31.8 \%$, $\mathrm{p}=0.0008)$.

Conclusion: A pre-ECMO lactate of $9.8 \mathrm{mmol} / \mathrm{L}$ or less may predict a favorable outcome at 6 months in ACS patients undergoing VA-ECMO. Further research aiming to improve the accuracy of predictions of reversibility in patients with high pre-ECMO lactate levels is essential.
\end{abstract}

Keywords: Cardiogenic shock, Acute coronary syndrome, Lactic acid, Extracorporeal membrane oxygenation

\section{Introduction}

Cardiogenic shock (CS) is a clinical condition involving inadequate tissue perfusion due to cardiac dysfunction. The definition includes the following hemodynamic parameters: persistent hypotension (systolic blood pressure $<90 \mathrm{~mm} \mathrm{Hg}$ or mean arterial blood pressure $30 \mathrm{~mm} \mathrm{Hg}$ low- er than baseline) with a severe reduction in the cardiac in$\operatorname{dex}\left(<1.8 \mathrm{~L} / \mathrm{min} / \mathrm{m}^{2}\right.$ without support or $<2$ to $2.2 \mathrm{~L} / \mathrm{min} / \mathrm{m}^{2}$ with support) and adequate or elevated filling pressures [1]. Some patients with CS are resistant to medical treatment, and in that case, extracorporeal membrane oxygenation (ECMO) provides practical cardiopulmonary support for myocardial recovery [2]. In addition, ECMO can be used as 
a "bridge to decision" in patients with acute and rapidly deteriorating heart failure or CS to stabilize hemodynamics, recover end-organ function, and enable an evaluation of the possibility of either heart transplantation or a more durable ventricular assist device [3].

ECMO is an important rescue therapy for severe CS, but mortality and morbidity remain high. The Survival After Veno-Arterial ECMO (SAVE) score may be the most popular tool to predict survival for patients receiving ECMO for refractory CS [4]. The SAVE score consists of several preECMO parameters: age, weight, diagnosis, chronic renal failure, acute pre-ECMO organ failure, peak inspiratory pressure, duration of intubation, cardiac arrest (CA), pulse pressure, diastolic pressure, and $\mathrm{HCO}_{3}^{-}$level [4]. This tool has been regarded as a complex model that includes many variables and numerous categories, necessitating an electronic calculation.

Lactate is formed from pyruvate in the cytosol as part of glycolysis [5]. Raised blood lactate concentrations in critically ill patients are frequently viewed as evidence of tissue hypoxia (type A hyperlactatemia). This tissue hypoxia has been widely believed to be a major cause of organ failure and mortality. Moreover, changes in lactate concentration over time during an intervention have been proposed as a treatment endpoint [6-8].

ECMO patients often die in a worsening condition after discharge from hospital or 1 month after ECMO initiation. However, most previous studies focused on predictive value of serum lactate levels for short-term outcomes, such as in-hospital or 1-month mortality, in ECMO patients. The purpose of our study was to evaluate the effect of pre-ECMO lactate levels on 6-month survival in patients with acute coronary syndrome (ACS) undergoing ECMO.

\section{Methods}

\section{Patient selection}

We retrospectively collected information on CS or CA patients who underwent veno-arterial (VA) ECMO from January 2015 to June 2020 at Chonnam National University Hospital. CS was diagnosed based on the 2016 European Society of Cardiology guideline (systolic blood pressure $<90 \mathrm{~mm} \mathrm{Hg}$ with appropriate fluid resuscitation with clinical and laboratory evidence of end-organ damage, such as cold extremities, oliguria, narrow pulse pressure, metabolic acidosis, elevated serum lactate, and elevated serum creatinine) [2]. VA ECMO was indicated for CA or refractory CS patients with tissue hypoperfusion, reduced left ventricular ejection fraction, and hypotension resistant to high-dose vasopressors or inotropic agents. In this period, 293 patients underwent VA-ECMO. Moreover, 148 adult patients were diagnosed with CA or refractory CS following ACS. Patients meeting the following criteria were excluded from the study: other causes of VA-ECMO support (such as post-cardiotomy shock, septic shock, and other undifferentiated origins of shock), age $\leq 18$ years, total ECMO duration $<12$ hours, and the availability of insufficient laboratory data. We divided all patients into survivors and nonsurvivors at 6 months, regardless of ECMO weaning. Clinical data were compared between the 2 groups.

\section{Extracorporeal membrane oxygenation protocol}

The cannulation technique and management strategy have been described in detail in previous studies [9]. In short, all ECMO-related procedures were performed under sterilized conditions with chlorhexidine. A polymethyl pentene fiber oxygenator (Maquet Inc., Hirrlingen, Germany) was applied for gas exchange in the extracorporeal circulation machines. Unfractionated heparin was infused during ECMO circulation. A multidisciplinary ECMO team determined the initiation of ECMO support based on the patient's characteristics, disease progression, and organ failure. We applied a peripheral cannulation strategy to all patients. Antibiotics were not routinely used unless there was distinct evidence of infection during ECMO support.

\section{Revascularization strategy}

The revascularization strategy in patients with ACS complicated by CA or CA who have single-vessel disease is an emergent percutaneous coronary intervention for the culprit artery. In cases of multivessel disease, we performed culprit-only revascularization in nearly all cases based on the results of the CULPRIT-SHOCK trial $[10,11]$. In ACS patients undergoing VA-ECMO for profound CS or CA, we usually revascularized only the culprit artery in patients dependent on VA-ECMO in the index procedure. Even though the decision and timing for revascularization of the non-culprit artery is at the operators' discretion, it was recommended to perform revascularization of a non-culprit artery with large myocardial blood supply territory, such as the left main or proximal left anterior descending artery, in the index procedure. If patients had left main or multivessel disease with good antegrade flow, coronary artery bypass graft was also performed as emergent or urgent surgery according to the operators' discretion. 


\section{Data collection}

All clinical variables of patients, including clinical and laboratory data before and during the ECMO period, were retrospectively collected. Pre-ECMO laboratory data were collected within 2 hours before ECMO onset. In-hospital mortality was assessed according to whether the death occurred during hospitalization, and 6-month mortality was calculated after 6 months of ECMO initiation. Serum lactate levels were measured from arterial blood gas analysis (GEM Premier 3000 system; Werfen Company, Bedford, MA, USA).

\section{Statistical analyses}

The results are expressed as mean values \pm standard devi- ation for continuous variables and as frequency and percentages (\%) for categorical variables. We used the independent t-test or chi-square test to compare continuous variables or categorical variables, respectively, according to 6-month survival. Cox proportional-hazards models were used to evaluate the univariable and multivariable hazard ratios (HRs) for 6-month mortality with a linear combination of pre-ECMO covariates that were confirmed to be statistically significant through the independent $\mathrm{t}$-test and chi-square test. A candidate variable with a univariate p-value $\leq 0.05$ was retained in the multivariable model. Receiver operating characteristic (ROC) curve analysis was done to identify the cut-off value of pre-ECMO lactate levels related to 6-month mortality with the DeLong test. The area under the ROC curve (AUC) was compared between pre-ECMO lactate levels and SAVE scores with and with-

Table 1. Patients' characteristics before ECMO support and a comparison between survivors and non-survivors defined in terms of 6-month mortality

\begin{tabular}{|c|c|c|c|c|}
\hline Characteristic & Total patients $(\mathrm{N}=148)$ & Survivors $(\mathrm{N}=68)$ & Non-survivors $(\mathrm{N}=80)$ & $\mathrm{p}$-value \\
\hline Age (yr) & $66.0 \pm 10.5$ & $65.0 \pm 10.8$ & $66.8 \pm 10.3$ & 0.323 \\
\hline Age $>80 \mathrm{yr}$ & $10(6.8)$ & $4(5.9)$ & $6(7.5)$ & 0.950 \\
\hline Body mass index $\left(\mathrm{kg} / \mathrm{m}^{2}\right)$ & $23.5 \pm 2.8$ & $23.8 \pm 3.0$ & $23.3 \pm 2.6$ & 0.282 \\
\hline \multicolumn{5}{|l|}{ Underlying disease } \\
\hline Hypertension & $84(56.8)$ & $40(58.8)$ & $44(55.0)$ & 0.763 \\
\hline Previous AMI & $25(16.9)$ & $15(22.1)$ & $10(12.5)$ & 0.185 \\
\hline Dyslipidemia & $16(10.8)$ & $9(13.2)$ & $7(8.8)$ & 0.542 \\
\hline Heart failure & $6(4.1)$ & $2(2.9)$ & $4(5.0)$ & 0.830 \\
\hline Diabetes mellitus & $68(45.9)$ & $33(48.5)$ & $35(43.8)$ & 0.677 \\
\hline Chronic kidney disease & $6(4.1)$ & $1(1.5)$ & $5(6.2)$ & 0.293 \\
\hline Cerebrovascular accident & $14(9.5)$ & $5(7.4)$ & $9(11.2)$ & 0.599 \\
\hline Smoking & $70(47.3)$ & $28(41.2)$ & $42(52.5)$ & 0.226 \\
\hline \multicolumn{5}{|l|}{ Laboratory data } \\
\hline White blood count $\left(10^{3} / \mathrm{mm}^{3}\right)$ & $13.8 \pm 5.5$ & $14.7 \pm 6.2$ & $13.2 \pm 5.0$ & 0.130 \\
\hline Hemoglobin (g/dL) & $12.0 \pm 2.5$ & $12.4 \pm 2.4$ & $11.8 \pm 2.6$ & 0.171 \\
\hline Platelets $\left(10^{3} / \mathrm{mm}^{3}\right)$ & $214.2 \pm 86.3$ & $226.4 \pm 89.2$ & $203.3 \pm 82.9$ & 0.126 \\
\hline Lactate $(\mathrm{mmol} / \mathrm{L})$ & $8.6 \pm 5.0$ & $7.2 \pm 4.3$ & $9.8 \pm 5.3$ & 0.002 \\
\hline Troponin $(\mathrm{ng} / \mathrm{mL})$ & $75.0 \pm 92.9$ & $59.9 \pm 79.4$ & $89.3 \pm 102.5$ & 0.065 \\
\hline $\mathrm{HCO}_{3}^{-}(\mathrm{mmol} / \mathrm{L})$ & $15.4 \pm 6.0$ & $16.8 \pm 5.2$ & $14.2 \pm 6.3$ & 0.008 \\
\hline \multicolumn{5}{|l|}{ CPR information } \\
\hline ED ECMO & $60(40.5)$ & $26(38.2)$ & $34(42.5)$ & 0.720 \\
\hline ECPR & $64(43.2)$ & $17(25.0)$ & $47(58.8)$ & $<0.001$ \\
\hline CPR to ECMO time (min) & $36.9 \pm 23.5$ & $35.1 \pm 24.7$ & $37.6 \pm 23.2$ & 0.708 \\
\hline Bystander CPR & $16(10.8)$ & $4(5.9)$ & $12(15.0)$ & 0.130 \\
\hline Witness arrest & $19(12.8)$ & $5(7.4)$ & $14(17.5)$ & 0.111 \\
\hline SAVE score & $-7.6 \pm 5.1$ & $-5.7 \pm 5.1$ & $-9.3 \pm 4.6$ & $<0.001$ \\
\hline Shock to ECMO time (hr) & $4.9 \pm 7.5$ & $3.9 \pm 5.3$ & $5.3 \pm 8.8$ & 0.24 \\
\hline MV before ECMO (hr) & $9.5 \pm 13.9$ & $28(41.2)$ & $18(22.5)$ & 0.023 \\
\hline 2DE EF (\%) & $32.0 \pm 14.8$ & $31.0 \pm 14.6$ & $33.6 \pm 15.3$ & 0.438 \\
\hline
\end{tabular}

Values are presented as mean \pm standard deviation or number $(\%)$.

ECMO, extracorporeal membrane oxygenation; AMI, acute myocardial infarction; CPR, cardiopulmonary resuscitation; ED, emergency department; ECPR, extracorporeal cardiopulmonary resuscitation; SAVE, Survival After Veno-Arterial Extracorporeal Membrane Oxygenation; MV, mechanical ventilation; 2DE EF, 2-dimensional echocardiography ejection fraction. 
out a history of extracorporeal cardiopulmonary resuscitation (ECPR). Kaplan-Meier curves and the log-rank test were used to assess time to death from the date of ECMO initiation to 180-day survival for groups stratified according to the cut-off value of pre-ECMO lactate for 6-month mortality. We used MedCalc statistical software ver. 17.9.7 (MedCalc Software bvba, Ostend, Belgium) for the statistical analysis. A generalized additive model was used to evaluate the nonlinear regression between the biomarkers and mortality using Stata ver. 17.0 (StataCorp., College Station, TX, USA). In all analyses, p-values $<0.05$ were considered to indicate statistical significance.

\section{Ethics statement}

The Institutional Review Board validated the research. All data from this study were obtained without the consent of patients due to the retrospective nature of this work (IRB no., CNUH-2020-023).

\section{Results}

\section{Patient demographics and outcomes of extracorporeal membrane oxygenation support}

We evaluated 148 patients with CA or refractory CS following ACS who were treated with VA-ECMO. The mean

Table 2. Analysis of clinical information related to coronary revascularization during ECMO support

\begin{tabular}{|c|c|c|c|c|}
\hline Variable & Total $(\mathrm{N}=148)$ & Survivors $(\mathrm{N}=68)$ & Non-survivors $(\mathrm{N}=80)$ & $\mathrm{p}$-value \\
\hline \multicolumn{5}{|l|}{ Diagnosis } \\
\hline ST-segment elevation myocardial infarction & $80(54.1)$ & $36(52.9)$ & $44(55.0)$ & 0.932 \\
\hline Left main coronary artery lesion & $49(33.1)$ & $22(32.4)$ & $27(33.8)$ & 0.996 \\
\hline Initial revascularization & & & & 0.099 \\
\hline Percutaneous coronary intervention & $127(85.8)$ & $54(42.5)$ & $73(57.5)$ & \\
\hline Coronary artery bypass grafta) & $14(9.5)$ & $10(71.4)$ & $4(28.6)$ & \\
\hline No revascularization ${ }^{\text {b) }}$ & $7(4.7)$ & $4(57.1)$ & $3(42.9)$ & \\
\hline Definite stent thrombosis & $10(6.8)$ & $5(7.4)$ & $5(6.2)$ & 1.000 \\
\hline
\end{tabular}

Values are presented as or number (\%).

$\mathrm{ECMO}$, extracorporeal membrane oxygenation.

a) Patients who underwent veno-arterial-ECMO in the preoperative period. not related to post-cardiotomy shock. ${ }^{\text {b)}}$ Clinically relevant with acute coronary syndrome, but spasm on coronary artery angiogram findings.

Table 3. Comparison of ECMO outcomes between survivors and non-survivors defined in terms of 6-month mortality

\begin{tabular}{|c|c|c|c|c|}
\hline Variable & Total $(\mathrm{N}=148)$ & Survivors $(\mathrm{N}=68)$ & Non-survivors $(\mathrm{N}=80)$ & p-value \\
\hline \multicolumn{5}{|l|}{ Microbiology data } \\
\hline Blood culture (bacteria) & $36(24.3)$ & $21(30.9)$ & $15(18.8)$ & 0.128 \\
\hline Blood culture (fungus) & $3(2.0)$ & $2(2.9)$ & $1(1.2)$ & 0.887 \\
\hline Respiratory culture & $58(39.2)$ & $32(47.1)$ & $26(32.5)$ & 0.101 \\
\hline \multicolumn{5}{|l|}{ Complications } \\
\hline Targeted temperature management & $8(5.4)$ & 0 & $8(10.0)$ & 0.021 \\
\hline Cerebral infarction & $6(4.1)$ & $2(3.0)$ & $4(5.0)$ & 0.844 \\
\hline Cerebral hemorrhage & $3(2.0)$ & $1(1.5)$ & $2(2.5)$ & 1.000 \\
\hline Acute brain injury & $4(2.7)$ & 0 & $4(5.0)$ & 0.178 \\
\hline Acute kidney injury & $59(40.1)$ & $42(62.7)$ & $46(57.5)$ & 0.638 \\
\hline Continuous renal replacement therapy & $68(46.3)$ & $23(34.3)$ & $45(56.2)$ & 0.638 \\
\hline \multicolumn{5}{|l|}{ ECMO outcome } \\
\hline ECMO duration (day) & $5.8 \pm 6.5$ & $5.7 \pm 5.5$ & $5.9 \pm 7.2$ & 0.804 \\
\hline ECMO weaning success & $79(53.4)$ & $66(97.1)$ & $13(16.2)$ & $<0.001$ \\
\hline Hospital stays (day) & $37.4 \pm 101.1$ & $49.3 \pm 65.0$ & $27.3 \pm 123.3$ & 0.169 \\
\hline 1-Month death & $72(48.6)$ & & & \\
\hline 6-Month death & $80(54.1)$ & & & \\
\hline
\end{tabular}

Values are presented as number (\%) or mean \pm standard deviation.

$\mathrm{ECMO}$, extracorporeal membrane oxygenation. 
patient age at the time of ECMO support was $66.0 \pm 10.5$ years, and 116 patients (78.4\%) were men. Among them, 25 patients (16.9\%) had a history of previous acute myocardial infarction. The duration of shock before ECMO initiation was $4.9 \pm 7.5$ hours. There were significant differences in 6 -month mortality between the 2 groups in pre-ECMO lactate and $\mathrm{HCO}_{3}{ }^{-}$levels, the duration of mechanical ventilation (MV) before ECMO, and ECPR. Patient characteristics before ECMO support and the results of the risk factor analysis for 6-month mortality are summarized in Table 1. Clinical information on coronary revascularization before and after ECMO support is presented in Table 2.

In the analysis of clinical outcomes, the mean duration of ECMO support was $5.8 \pm 6.5$ days, and the rate of successful ECMO weaning was $53.4 \%$. There were no statistically differences in these variables between survivors and non-survivors. Sixty-eight patients (45.9\%) were discharged from the hospital alive and reached a 180-day follow-up after ECMO initiation. Renal replacement therapy was applied during ECMO support in 68 patients (46.3\%). Eight patients (5.4\%) underwent target temperature management (TTM), but all of these patients died after TTM. All causes of acute brain injury (cerebral infarction, hemorrhage, hypoxic ischemic brain injury) were diagnosed in fewer than $5 \%$ of patients. The outcomes of ECMO support are summarized in Table 3.

\section{Cox proportional-hazards regression for covariates associated with 6-month mortality}

The Cox proportional-hazards regression estimated the hazard for 6-month death according to pre-ECMO covariates that were confirmed to be statistically significant through the independent $\mathrm{t}$-test and chi-square test: pre-ECMO lactate, pre-ECMO $\mathrm{HCO}_{3}^{-}$, $\mathrm{MV}$ duration before ECMO initiation, and ECPR history. The Cox model showed that pre-ECMO lactate level and MV duration before ECMO application were significantly associated with 6-month mortality (HR, 1.19; 95\% CI, 1.06-1.34; $\mathrm{p}=0.003$ and HR, 1.04; 95\% CI, 1.02-1.07; $\mathrm{p}=0.002$, respectively) (Table 4).

\section{Evaluation of the pre-extracorporeal membrane oxygenation lactate level as a survival predictor at 6 months}

The AUC of pre-ECMO lactate was 0.64 (standard error $[\mathrm{SE}]=0.05 ; 95 \% \mathrm{CI}, 0.56-0.72 ; \mathrm{p}=0.002$; cut-off value $=9.8$ ) (Fig. 1). We compared the AUC between the pre-ECMO lactate level and SAVE score for predicting 6-month mortality. A subgroup analysis was also performed based on ECPR history, which was one of the 6-month survival predictors, and the following results were obtained. In the analysis of all included patients, although the AUC of the SAVE score (0.69) was slightly higher than that of the

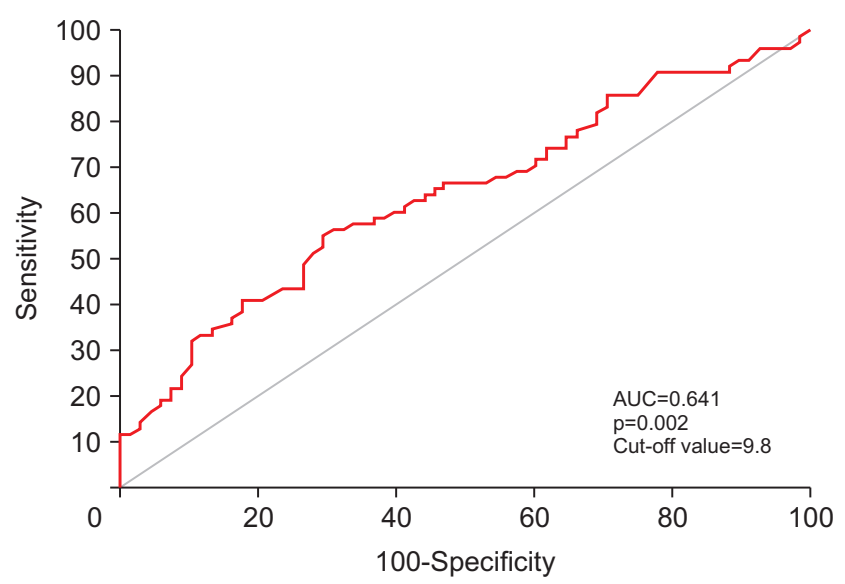

Fig. 1. Receiver operating characteristic (ROC) curve of pre-extracorporeal membrane oxygenation (ECMO) lactate levels for the prediction of 6 -month mortality. The area under the ROC curve of pre-ECMO lactate levels was 0.64 (standard error=0.05; $95 \%$ confidence interval, $0.56-0.72 ; p=0.002$, cut-off value $=9.8$ $\mathrm{mmol} / \mathrm{L})$. AUC, area under ROC curve.

Table 4. Cox proportional-hazards regression with pre-ECMO covariates based on 6-month mortality

\begin{tabular}{|c|c|c|c|c|}
\hline \multirow{2}{*}{ Variable } & \multicolumn{2}{|c|}{ Univariable analyses $^{\text {a) }}$} & \multicolumn{2}{|c|}{ Multivariable analyses ${ }^{\text {b) }}$} \\
\hline & $\operatorname{HR}(95 \% \mathrm{Cl})$ & $\mathrm{p}$-value & $\operatorname{HR}(95 \% \mathrm{Cl})$ & $\mathrm{p}$-value \\
\hline Lactate (pre-ECMO) & $1.096(1.045-1.149)$ & $<0.001$ & $1.210(1.064-1.376)$ & 0.004 \\
\hline ECPR history & $2.968(1.725-4.221)$ & $<0.001$ & $0.408(0.105-1.584)$ & 0.195 \\
\hline $\mathrm{HCO}_{3}^{-}$ & $0.940(0.904-0.978)$ & 0.002 & $1.040(0.923-1.172)$ & 0.519 \\
\hline MV before ECMO & $1.034(1.007-1.061)$ & 0.012 & $1.046(1.017-1.076)$ & 0.002 \\
\hline
\end{tabular}

ECMO, extracorporeal membrane oxygenation; $\mathrm{HR}$, hazard ratio; $\mathrm{Cl}$, confidence interval; $\mathrm{ECPR}$, extracorporeal cardiopulmonary resuscitation; $\mathrm{MV}$, mechanical ventilation.

a) Combination of pre-ECMO covariates, which were confirmed to be statistically significant through the independent t-test and chi-square test, were included in the univariable analysis. ${ }^{\text {b) }}$ Candidate variables with a univariate $p$-value $\leq 0.05$ were retained in the multivariable model. 
pre-ECMO lactate (0.65) in predicting the 6-month survival probability, the AUCs did not show a statistically significant difference (difference between areas $=0.06 ; \mathrm{SE}=0.06$; 95\% CI, 0.06-0.17; $\mathrm{p}=0.342$ ) (Fig. 2A). In the subgroup analysis of non-ECPR patients, there was no statistically significant difference in the AUC (difference between areas $=0.04 ; \mathrm{SE}=0.08 ; 95 \% \mathrm{CI}, 0.13-0.20 ; \mathrm{p}=0.643$ ) (Fig. 2B). However, in the subgroup analysis of ECPR patients, the AUC did show a statistically significant difference (difference between areas $=0.2$; $\mathrm{SE}=0.09,95 \% \mathrm{CI}, 0.02-0.38$; $\mathrm{p}=$ 0.031) (Fig. 2C). These results confirmed that the pre-ECMO lactate level was not inferior to the SAVE score in predicting 6-month survival, and that the pre-ECMO lactate level had low efficacy in predicting survival in patients who underwent ECPR. Additional nonlinear regression testing showed no significant relationships between preECMO lactate or the SAVE score and mortality risk ( $\mathrm{p}=$ 0.450 for pre-ECMO lactate, $\mathrm{p}=0.160$ for the SAVE score) (Supplementary Fig. 1). The impact of the pre-ECMO lactate level on 6-month mortality was estimated by Kaplan-Meier survival analysis in patients who had pre-EC-
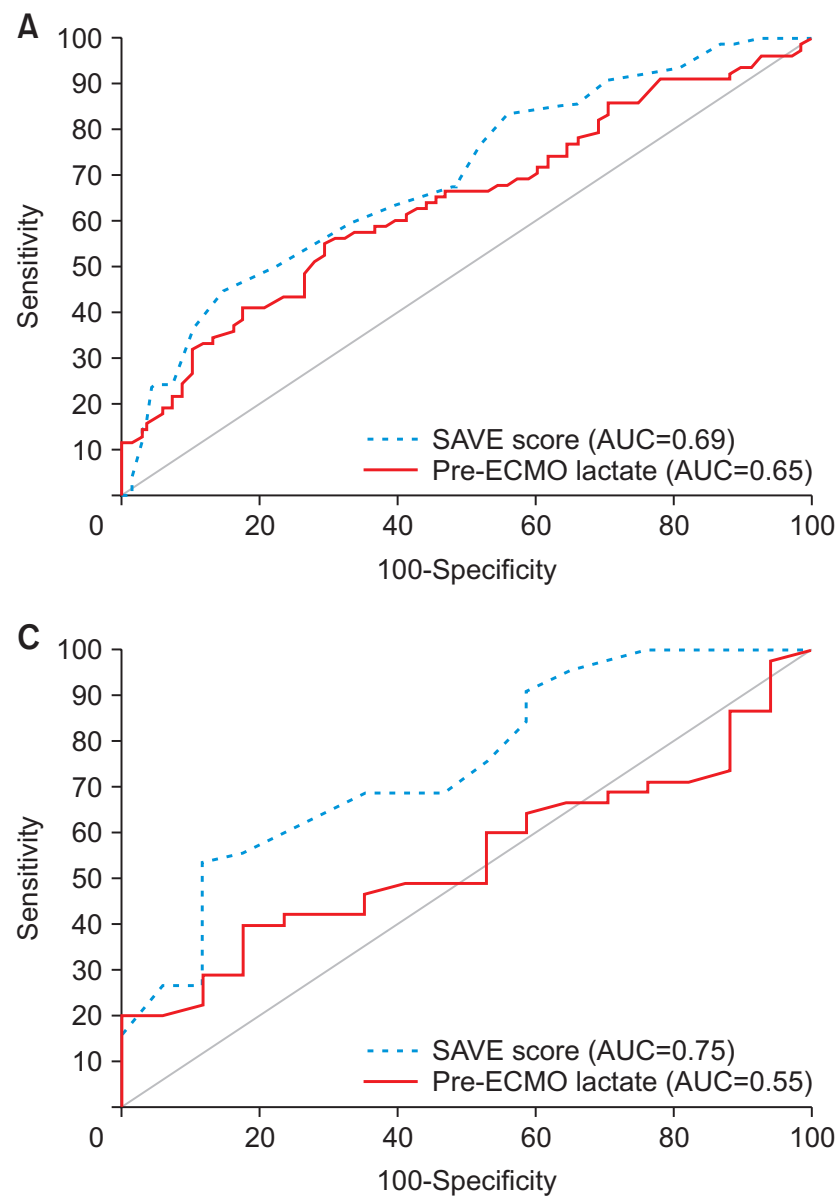

MO lactate levels of more than $9.8 \mathrm{mmol} / \mathrm{L}$ and less than $9.8 \mathrm{mmol} / \mathrm{L}$. The patients with pre-ECMO lactate levels of more than $9.8 \mathrm{mmol} / \mathrm{L}$ had a lower survival probability $(\mathrm{p}<0.0008)$ (Fig. 3).

\section{Discussion}

According to a recent Extracorporeal Life Support Organization report, approximately $45 \%$ of patients treated with VA-ECMO are discharged alive, but some are known to die after discharge [12]. The mortality rate after VA-ECMO remains high due to the underlying disease that has not been cured and the worsening of ECMO-related complications. As the clinical outcomes of ECMO support (especially VA-ECMO) are still unsatisfactory, even after the intensive use of large-scale medical resources, so accurate evaluation is essential from the first initiation of ECMO.

The SAVE score is the most popular tool to predict successful ECMO weaning based on the pre-ECMO situation, and its usefulness has been reported in several studies $[4,13,14]$. Chen et al. [14] reported that serum lactate levels

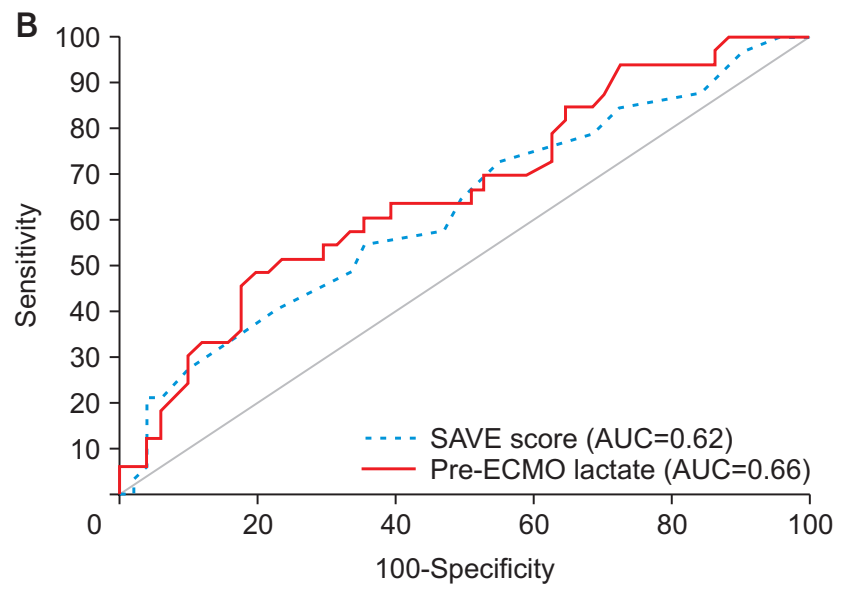

Fig. 2. Comparison of receiver operating characteristic (ROC) curves between pre-extracorporeal membrane oxygenation (ECMO) lactate levels and Survival After Veno-Arterial Extracorporeal Membrane Oxygenation (SAVE) scores for the prediction of 6-month mortality with and without extracorporeal cardiopulmonary resuscitation (ECPR). (A) Comparison of ROC curves in all patients (difference between areas $=0.06$; standard error $[S E]=0.06 ; 95 \%$ confidence interval $[\mathrm{Cl}], 0.06-0.17 ; \mathrm{p}=0.342$ ). (B) Comparison of ROC curves in non-ECPR patients (difference between areas $=0.03 ; \mathrm{SE}=0.08 ; 95 \% \mathrm{Cl}, 0.13-0.20 ; \mathrm{p}=0.643)$. (C) Comparison of ROC curves in ECPR patients (difference between areas $=0.2 ; \mathrm{SE}=0.09 ; 95 \% \mathrm{Cl}, 0.02-0.38 ; \mathrm{p}=0.031)$. AUC, area under ROC curve. 


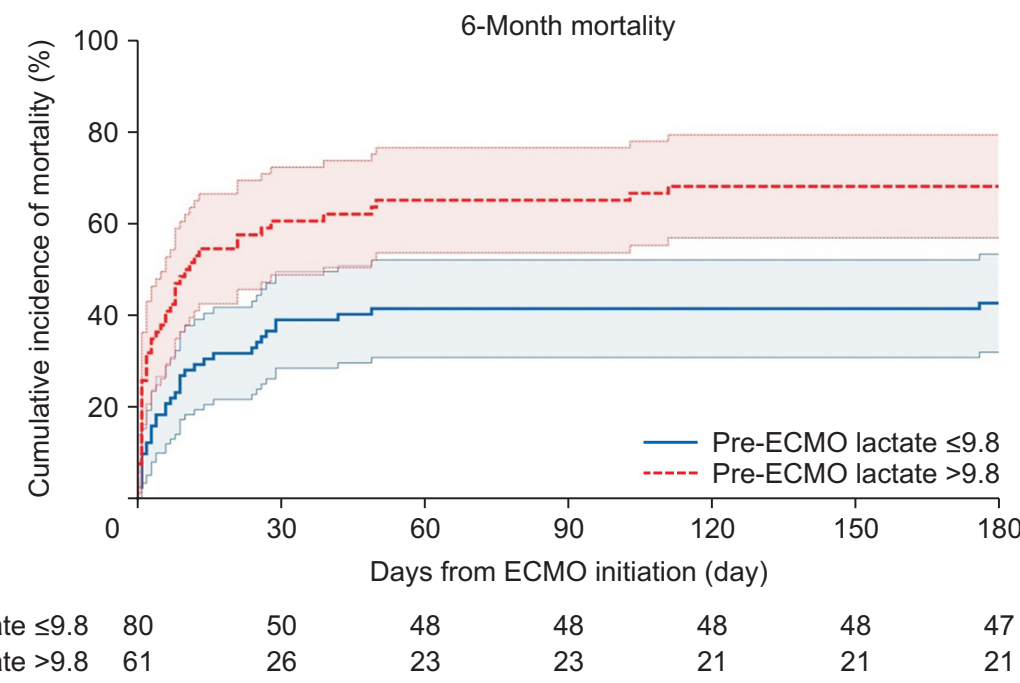

26
Fig. 3. Survival analysis with cumulative mortality incidence from the time of extracorporeal membrane oxygenation $(E C M O)$ initiation. Patients with pre-ECMO lactate levels of more than $9.8 \mathrm{mmol} / \mathrm{L}$ had a lower survival probability $(\mathrm{p}<0.0008)$ than patients with pre-ECMO lactate levels of less than $9.8 \mathrm{mmol} / \mathrm{L}$.
No. at risk

$\begin{array}{ll}\text { Pre-ECMO lactate } \leq 9.8 & 80 \\ \text { Pre-ECMO lactate }>9.8 & 61\end{array}$ and SAVE scores were both statistically significant predictors of mortality in Cox proportional-hazards regression. The AUC of lactate levels was superior to that of SAVE scores to predict 90-day mortality (serum lactate level= 0.79 , SAVE score $=0.73$ ) [14]. They proposed a combination of the blood lactate levels and the SAVE score, referred to as the modified SAVE score, although the lactate level alone showed sufficient clinical significance for differentiating the survival prognosis. As discussed earlier, the SAVE score has been regarded as a complex model that includes many variables and numerous categories.

From a historical viewpoint, many researchers have reported that increased lactic acid levels are a risk factor for death in critically ill patients with diverse diseases, including CS [8,15-17]. Several recent studies have investigated the use of lactate levels as a surrogate marker to evaluate end-organ hypoperfusion and survival after ECMO weaning $[14,18,19]$. In previous studies, pre-ECMO lactate levels and/or lactate metabolism after ECMO initiation had a strong effect on viability, and specific cut-off values of serum lactate have been suggested as thresholds for predicting short-term mortality, such as in-hospital or 1-month mortality. However, previous research contained little information on the association between pre-ECMO lactate levels and mid- to long-term outcomes after hospital discharge. For this reason, we set 6-month mortality as the primary outcome of our study, rather than a short-term outcome. As with the results of previous studies, serum lactate levels were a reliable predictor of 1-month and 6month mortality in patients who underwent V-A ECMO in our study. The best threshold value of pre-ECMO lactate levels was more than $9.8 \mathrm{mmol} / \mathrm{L}$ in the ROC curve analy- sis for 6-month survival in our results. Moreover, the Cox proportional-hazards ratio showed that pre-ECMO lactate levels were a robust predictor.

This study has some limitations. First, this single-center study enrolled a relatively small number of patients. Second, as a retrospective cohort study, a few patients were lost to follow-up, resulting in selection bias. Finally, we followed the general design of a clinical study performing a survival analysis. However, categorizing the study group into survivors and non-survivors was an intentional division, and this method may have also induced selection bias.

In conclusion, ACS patients who underwent VA-ECMO with lactate levels $>9.8 \mathrm{mmol} / \mathrm{L}$ prior to ECMO had significantly lower 6-month survival rates. However, the serum lactate level before ECMO alone is still limited as a predictor of survival, and its efficacy is further reduced when it is accompanied by serious risk factors such as ECPR. In the next study, a more accurate analysis of pre-ECMO lactate levels will be performed to predict survival after VA-ECMO for CS patients with high lactate levels.

\section{Conflict of interest}

No potential conflict of interest relevant to this article was reported.

\section{Funding}

This study was supported by the National Research Foundation of Korea (NRF-2019R1D1A3A03103899, NRF2021R1I1A3047390). 


\section{ORCID}

Eunchong Kim: https://orcid.org/0000-0002-1119-4125

Nodirbek Yuldashev Sodirzhon-Ugli:

https://orcid.org/0000-0002-9635-5978

Do Wan Kim: https://orcid.org/0000-0003-2262-2882

Kyo Seon Lee: https://orcid.org/0000-0001-7397-4680

Yonghwan Lim: https://orcid.org/0000-0002-2405-1676

Min-Chul Kim: https://orcid.org/0000-0001-6026-1702

Yong Soo Cho: https://orcid.org/0000-0001-8306-2298

Yong Hun Jung: https://orcid.org/0000-0001-8531-7039

Kyung Woon Jeung: https://orcid.org/0000-0002-3478-6967

Hwa Jin Cho: https://orcid.org/0000-0002-2458-8529

In Seok Jeong: https://orcid.org/0000-0002-2249-0667

\section{Supplementary materials}

Supplementary materials can be found via https://doi. org/10.5090/jcs.21.127. Supplementary Fig 1. Nonlinear regression test of mortality.

\section{References}

1. Vahdatpour C, Collins D, Goldberg S. Cardiogenic shock. J Am Heart Assoc 2019;8:e011991.

2. Ponikowski P, Voors AA, Anker SD, et al. 2016 ESC Guidelines for the diagnosis and treatment of acute and chronic heart failure: the Task Force for the diagnosis and treatment of acute and chronic heart failure of the European Society of Cardiology (ESC) developed with the special contribution of the Heart Failure Association (HFA) of the ESC. Eur Heart J 2016;37:2129-200.

3. Riebandt J, Haberl T, Mahr S, et al. Preoperative patient optimization using extracorporeal life support improves outcomes of INTERMACS level I patients receiving a permanent ventricular assist device. Eur J Cardiothorac Surg 2014;46:486-92.

4. Schmidt M, Burrell A, Roberts L, et al. Predicting survival after ECMO for refractory cardiogenic shock: the survival after veno-arterial-ECMO (SAVE)-score. Eur Heart J 2015;36:2246-56.

5. Garcia-Alvarez M, Marik P, Bellomo R. Sepsis-associated hyperlactatemia. Crit Care 2014;18:503.

6. Dellinger RP, Levy MM, Rhodes A, et al. Surviving Sepsis Campaign: international guidelines for management of severe sepsis and septic shock, 2012. Intensive Care Med 2013;39:165-228.

7. Sterling SA, Puskarich MA, Shapiro NI, et al. Characteristics and outcomes of patients with vasoplegic versus tissue dysoxic septic shock. Shock 2013;40:11-4.

8. Jansen TC, van Bommel J, Bakker J. Blood lactate monitoring in critically ill patients: a systematic health technology assessment. Crit Care Med 2009;37:2827-39.

9. Kim DW, Cho HJ, Kim GS, et al. Predictive value of procalcitonin for infection and survival in adult cardiogenic shock patients treated with extracorporeal membrane oxygenation. Chonnam Med J 2018; 54:48-54.

10. Thiele H, Akin I, Sandri M, et al. PCI strategies in patients with acute myocardial infarction and cardiogenic shock. N Engl J Med 2017;377:2419-32.

11. Thiele H, Akin I, Sandri M, et al. One-year outcomes after PCI strategies in cardiogenic shock. N Engl J Med 2018;379:1699-710.

12. Lorusso R, Shekar K, MacLaren G, et al. ELSO interim guidelines for venoarterial extracorporeal membrane oxygenation in adult cardiac patients. ASAIO J 2021;67:827-44.

13. Vasques F, Romitti F, Gattinoni L, Camporota L. How I wean patients from veno-venous extra-corporeal membrane oxygenation. Crit Care 2019;23:316.

14. Chen WC, Huang KY, Yao CW, et al. The modified SAVE score: predicting survival using urgent veno-arterial extracorporeal membrane oxygenation within 24 hours of arrival at the emergency department. Crit Care 2016;20:336.

15. Broder G, Weil MH. Excess lactate: an index of reversibility of shock in human patients. Science 1964;143:1457-9.

16. Luft D, Deichsel G, Schmülling RM, Stein W, Eggstein M. Definition of clinically relevant lactic acidosis in patients with internal diseases. Am J Clin Pathol 1983;80:484-9.

17. Stacpoole PW, Wright EC, Baumgartner TG, et al. Natural history and course of acquired lactic acidosis in adults. DCA-Lactic Acidosis Study Group. Am J Med 1994;97:47-54.

18. Slottosch I, Liakopoulos O, Kuhn E, et al. Lactate and lactate clearance as valuable tool to evaluate ECMO therapy in cardiogenic shock. J Crit Care 2017;42:35-41.

19. Fux T, Holm M, Corbascio M, Lund LH, van der Linden J. Venoarterial extracorporeal membrane oxygenation for postcardiotomy shock: risk factors for mortality. J Thorac Cardiovasc Surg 2018;156:1894902. 